\title{
EMPIRIC VERSUS ENTRAINED CAVOTRICUSPID ISTHMUS LINE ABLATION IN CONGENITAL HEART DISEASE: A SINGLE CENTRE EXPERIENCE
}

\author{
Sawhney $\mathrm{V}^{1,2}$, McLellan $\mathrm{A}^{1}$, Chatha $\mathrm{S}^{1}$, Perera $\mathrm{D}^{1}$, Aderonke $\mathrm{A}^{1}$, Juno $\mathrm{S}^{1}$, \\ Whittaker-Axon $\mathrm{S}^{1,2}$, Daw $\mathrm{H}^{1,2}$, Garcia $\mathrm{J}^{1}$, Lambiase $\mathrm{P}^{1}$, Bhan $\mathrm{A}^{2}$, Cullen $\mathrm{S}^{2}$, \\ VonKlemperer $\mathrm{K}^{2}$, Walker $\mathrm{F}^{2}$, Pandya $\mathrm{B}^{2}$, Lowe $\mathrm{M}^{1,2}$, Ezzat $\mathrm{V}^{1,2}$ \\ 1. Department of Arrhythmia Services, Barts Heart Centre, St Bartholomew's \\ Hospital, London, United Kingdom \\ 2. Grown-up Congenital Heart Disease Services, Barts Heart Centre, St \\ Bartholomew's Hospital, London, United Kingdom
}

"Corresponding Author: Vinit Sawhney

Department of Arrhythmia services,

Barts Heart Centre, St Bartholomew's Hospital,

London EC1A 7BE, UK.

Tel 4420376 58682, Fax 442034656483

Email: sawhneyvin@googlemail.com

Conflict of interest: Nil 


\section{ABSTRACT}

Background: Catheter ablation for supraventricular tachycardia (SVT) in adults with congenital heart disease (ACHD) is an important therapeutic option. Cavotricuspid isthmus (CTI) dependent intra-atrial re-entrant tachycardia (IART) is common. However, induction of sustained tachycardia at the time of ablation is not always possible. We hypothesised that performing an empiric CTI line in case of noninducibility leads to good outcomes.

Objectives: Long-term outcomes of empiric versus entrained CTI ablation in CHD patients were examined.

Methods: Retrospective, single-centre, case-control study over seven years. Arrhythmia free survival post empiric versus entrained CTI ablation were compared. Results: 87 CTI ablations were performed in 85 ACHD patients between 2010 and 2017. The mean age of the cohort was 43 years and $48 \%$ were men. Underlying etiology included ASD (31\%), VSD (11.4\%), AVSD (9.1\%), AVR (4.8\%), Fallot's tetralogy (18.4\%), Ebstein's anomaly (2.3\%), Fontan surgery $(9.2 \%)$ and Mustard/Senning repair (13.8\%). CTI dependent IART was entrained in 59 patients whereas it was non-inducible in 28 . The latter had an empiric CTI-line ablation. $57 \%$ of procedures were performed without a general anaesthetic. There were no procedural complications. There was no significant difference in the mean procedure and fluoroscopy times between the groups (Empiric vs Entrained CTI; 169.1 vs 183.3 and 28.1 vs $19.9 \mathrm{~min})$. After a mean follow-up of 21 months, arrhythmia-free survival was $64.3 \%$ versus $72.8 \%$ ( $p$-value 0.44 ) in the empiric and entrained CTI groups. Conclusion: Long-term outcomes after empiric and entrained CTI line ablation for IART ACHD patients are comparable. This is a safe and effective therapeutic option. In the case of non-inducibility of IART, an empiric CTI line ablation should be performed in this cohort. 
Keywords: intra-atrial re-entrant tachycardia, adult congenital heart disease, empiric cavo-tricuspid isthmus ablation

\section{CONDENSED ABSTRACT}

Intra-atrial re-entrant tachycardia (IART) is common in patients with congenital heart disease. Inability to induce sustained, haemodynamically stable tachycardia poses a major challenge to define ablation targets. We report that in such patients, performing an empiric cavo-tricuspid isthmus (CTI) line has good arrhythmia free survival rates which are not different to those in whom ablation is performed following entrainment of IART at CTI.

\section{WHAT'S NEW?}

- Catheter ablation for cavo-tricuspid isthmus (CTI) dependent intra-atrial reentrant tachycardia (IART) in adults with congenital heart disease is a safe and effective therapeutic option.

- Long-term outcomes (arrhythmia free survival) after empiric versus entrained CTI dependent IART are comparable.

- In case of non-inducibility of IART in CHD patients, an empiric CTI line ablation should be performed. 


\section{INTRODUCTION}

In the growing number of adult patients with congenital heart disease (ACHD), supraventricular tachycardia is the commonest cause of morbidity and hospital admissions. ${ }^{1-4}$ The prevalence increases with time since surgery, complexity of underlying defect, type of repair and older age at surgery. ${ }^{5}$ Given the poor haemodynamic reserve of these patients, urgent restoration of sinus rhythm by electric cardioversion remains the first line treatment option. ${ }^{6,7}$ Catheter ablation has emerged as a highly successful alternative therapeutic option in most cases, either alone or in combination with ongoing drug treatment. ${ }^{8,9}$

In vast majority of patients, intra-atrial re-entry (IART) is the most frequent arrhythmia mechanism. IART develops on the basis of complex substrate including atrial incisions, sutures and patch material as well as atrial stretch, dilatation and fibrosis owing to haemodynamic drivers. ${ }^{5}$ This complex substrate is one of the major challenges in mapping and ablation of arrhythmias in ACHD patients. It is not uncommon for the arrhythmia to terminate or degenerate into atrial fibrillation preventing further mapping to identify the clinically relevant mechanism. Moreover, these patients might have multiple non-clinical arrhythmias or arrhythmias might be non-inducible or cause haemodynamic compromise, all of which add to the challenge of catheter ablation in this cohort. In such cases, an empiric cavo-tricuspid isthmus (CTI) line may be performed. However, the long-term outcomes of empiric ablation remain unknown.

We hypothesised that performing an empiric cavo-tricuspid isthmus (CTI) line ablation in case of non-inducibility leads to good outcomes. In this study we report our experience of catheter ablation of cavo-tricuspid isthmus dependent tachycardias in ACHD patients. We compared patients who underwent empiric versus entrained CTI line ablation in a case-control study to compare the safety and efficacy of both approaches. 


\section{METHODS}

\section{Study design:}

This was a retrospective, single centre, case-control study examining the long-term outcomes of empiric versus entrained CTI line ablation in CHD patients with rightsided supraventricular arrhythmias. Consecutive patients over 9 years were included. Patients having empiric ablation were compared to those undergoing ablation of entrained CTI dependent IART. Prior to the procedure, all patients gave written informed consent. All data presented were prospectively recorded in our departmental electrophysiology registry.

\section{Study patients:}

All patients with $\mathrm{CHD}$ and undergoing catheter ablation of the cavo-tricuspid isthmus (CTI) were included in the study. All patients were anticoagulated at the time of the procedure. A trans-oesophageal echocardiogram (TOE) was done if clinically indicated. Patients who had CTI dependent flutter induced and ablated were compared to those in which IART could not be induced and an empiric CTI line was done.

\section{Mapping and Ablation:}

Procedures were carried out either under intravenous moderate sedation or a general anaesthetic.

Vascular ultrasound was routinely used for obtaining venous/arterial access. Three punctures were performed for radiofrequency ablation. Arterial puncture was obtained if retrograde access to the cavo-tricuspid isthmus was required. Patients were heparinised if clinically indicated aiming for an ACT of $300-350$ seconds.

Patients underwent standard ablation procedures as directed by the operator. Attempt at arrhythmia induction was done in all patients using pacing manouevres in addition to drugs. For arrhythmia induction, atrial burst pacing was performed from the coronary sinus and right atrium, starting from $400 \mathrm{~ms}$ down to $190 \mathrm{~ms}$, with and 
without isoprenaline. Mapping systems used included CARTO (Biosense Webster, Diamond Bar, California, USA) and Precision NavX (Abbott Medical, St Paul, Minnesota, USA). Irrigated radiofrequency catheters were used in all cases.

Post-procedure, sheaths were removed and manual pressure applied to site of vascular access. In heparinised patients, heparin was reversed with protamine and a Femostop (St Jude Medical) was applied to the site of vascular access. A transthoracic echocardiogram was performed routinely to look for evidence of pericardial effusion. The oral anticoagulant was continued as normal post-procedure without omitting any doses. Most patients continued to be on an anti-arrhythmic for three months post-procedure.

\section{Follow up:}

Patients were kept in overnight and discharged home the following day. They were reviewed in clinic 3 months post ablation. Additional review of medical notes and electronic health records was carried out to ensure capture of all complications.

\section{Study end points:}

The primary end point of the study was a composite of long-term success (arrhythmia free survival on follow-up) of empiric versus entrained CTI ablation in the absence of major complications. Success was defined as resolution of symptoms on follow-up (minimum 3 months) and no documented clinical IART on holter monitor. Procedural complications and predictors of success were also examined.

Acute procedural success was defined as termination of arrhythmia, confirmation of bi-directional block across the CTI line and non-inducibility thereafter (in the absence of major complications) in the entrained group. Whereas in the empiric group confirmation of bi-directional block across CTI line post ablation in the absence of major complications constituted acute success. A major complication was 
defined as a procedural complication requiring intervention and delaying hospital discharge.

\section{Statistical analyses:}

Data were analysed on an intention-to-treat basis. All analyses were carried out using SAS version 9.3, statistical software. Continuous data were presented as mean \pm standard deviation or median (range) if not normally distributed. Categorical data were reported as a percentage. Continuous data were compared using unpaired ttest (if normally-distributed) and Mann-Whitney $U$ test if not normally-distributed. Categorical data were compared using chi-square test. A p-value of less than 0.05 was considered significant.

\section{RESULTS}

\section{Study patients:}

348 ablation procedures were performed in ACHD patients during the study period. $87 \mathrm{CTI}$ dependent atrial flutter ablations were performed in 85 patients with complex congenital heart disease over seven years $(2010-2017)$ at a single centre. The mean age of the cohort was 43 years and $48 \%$ of the patients were men. This cohort represented a range of underlying congenital heart disease. The commonest underlying etiologies, was atrial septal defect (ASD; $n=27,31 \%$ ), repaired Tetralogy of Fallot (ToF, $n=16,18 \%)$ and Mustard/Senning repair for Transposition of great arteries (TGA, $n=12,14 \%$ ). Others included ventricular septal defect (VSD, $n=10$, $12 \%$ ), atrioventricular septal defect (AVSD, $n=8,9 \%)$, Fontan circulation ( $n=8,9 \%)$, aortic valve replacement (AVR, $n=4,5 \%)$ and Ebstein's $(n=2,2 \%)$ - Figure 1. In 59 patients, we were able to induce a CTI dependent IART (Entrained Group) whereas in the remaining 28 patients no sustained clinical tachycardia could be induced and hence an empiric CTI line ablation was performed (Empiric Group). The two groups were well matched for clinical characteristics - Table 1. There was no significant difference in age, gender and RA diameter between the groups $(p=$ 
$0.10,0.84,0.65)$. The commonest underlying etiology was ASD in the Empiric group $(n=12,43 \%)$ and ToF in the Entrained group $(n=12,21 \%)$.

\section{Procedural data:}

A consultant electrophysiologist with specialist interest in grown up congenital heart disease was the primary operator for all procedures. Two operators (consultants) at a single centre performed these procedures. The vast majority of procedures were performed as an elective admission. However, nearly $20 \%$ of the patients in each group required an urgent/emergency procedure. Just over half of the patients in both groups had the procedure done under GA. The mean procedure and fluoroscopy times in the Entrained versus Empiric group were $183 \pm 57$ and $20 \pm 18$ min versus $169 \pm 53$ and $28 \pm 31$ min respectively - Table 2 .

\section{Study endpoints:}

\section{Primary endpoint}

There was no significant difference in the primary end point between the two groups (Entrained versus Empiric) - Figure 2. At a mean follow-up of 21 mths, arrhythmia free survival in the entrained versus empiric group was 75\% versus $69 \%$ ( $p$-value 0.55).

One patient in each group had a major complication. In the Entrained group, one patient with underlying ASD and CTI ablation presented with a pseudoaneurysm four days post ablation. This was injected with thrombin and patient discharged home the same day. The subsequent scans were unremarkable. Whereas, in the Empiric group, one patient with underlying secundum ASD undergoing CTI line and pulmonary vein isolation had a cardiac tamponade. A drop in blood pressure and significant pericardial effusion were noted after the transseptal sheath was placed in the left atrium (without needle deployment) via the unrepaired ASD. A pericardial drain was inserted and $500 \mathrm{ml}$ of blood stained fluid drained immediately. Heparin 
was reversed with protamine but no reversal agent was given for warfarin. No further recollection of pericardial fluid was seen on subsequent echocardiograms. No surgical intervention was required and the drain was removed $24 \mathrm{hrs}$ post procedure. The patient had a hospital stay of two days and made a full recovery. The incidence of major complications in the entire cohort was $2.2 \%$ and not significantly different between the groups $(p=0.46)$.

\section{Other complications}

Other reported complications included femoral haematoma $(n=1)$ and transient third degree AV block $(n=1)$ that subsequently recovered peri-procedurally. There was no reported procedural mortality in the entire cohort.

\section{Secondary endpoint - Acute Procedural Success}

Acute ablation success was similar in both groups \{Entrained versus Empiric; 95\% versus $93 \%, p=0.99)$.

\section{Freedom from Arrhythmia based on Underlying Etiology}

We also analysed the arrhythmia free survival at follow-up based on the complexity of underlying CHD. Figure 3 shows arrhythmia free survival based on type of $\mathrm{CHD}$ in both the Entrained and Empiric group. There was no significant difference between the groups.

\section{Multivariate analysis of Factors impacting Primary End Point}

A multivariate regression analyses was carried out to assess the impact of various factors on arrhythmia free survival. This analysis was performed for the entire cohort. There was no significant association between age, gender, RA diameter, type of $\mathrm{CHD}$, procedure time and ablation time and freedom from arrhythmia at a mean follow-up of 21 months ( $p$-values $0.09,0.63,0.21,0.71,0.72$ and 0.96 ). The 
individual factors and their odds ratios are shown in Table 3. The only factor that showed a weak association, albeit not reaching significance, with freedom from arrhythmia was the patient age. Younger chronological age at the time of ablation was more likely to be associated with a positive outcome.

\section{DISCUSSION}

The present study shows that there is no significant difference in the arrhythmia free survival in patients undergoing entrained versus empiric CTI line ablation. At a mean follow-up of 21 months, $75 \%$ patients in the entrained group and $69 \%$ patients in the empiric group had no recurrence of IART post ablation. Performing an empiric CTI line ablation, in the absence of induction of the documented clinical arrhythmia, results in good long-term arrhythmia free survival rates. The complexity of underlying CHD had no significant association with the ablation outcomes of CTI dependent flutter. Patients' age at the time of ablation had a weak correlation with arrhythmia free survival rates, although not achieving statistical significance.

The prevalence of $A C H D$ population is on the rise owing to advances in surgical interventions. ${ }^{10} \mathrm{~A}$ considerable mortality reduction in patients with $\mathrm{CHD}$ has been noted and survival into adulthood is now $>90 \%$ resulting in nearly twice as many adult patients with CHD than paediatric patients with $\mathrm{CHD} \cdot{ }^{11}$ As a result, patients with ACHD are faced with significant morbidities, amongst which cardiac arrhythmias have a considerable impact not only on their quality of life but also on their long-term survival. ${ }^{12}$ Based on the underlying congenital anomaly and surgical correction, nearly $50 \%$ of the patients with ACHD develop supraventricular arrhythmias. ${ }^{13}$ Catheter ablation for SVT in this cohort is an important therapeutic option attributable to frequently experienced drug refractoriness and possible haemodynamic deterioration and thrombus formation. However, this is not without challenges give the complex anatomy, potentially difficult access to chamber of 
interest, multiple arrhythmia mechanisms and a progressive haemodynamic substrate in this group of patients.

Intra-atrial re-entrant tachycardias (IART) are the commonest arrhythmias in ACHD patients. These are usually secondary to a re-entrant circuit around scarred tissues due to atriotomies, patches or other natural or surgically created barriers to electrical conduction. ${ }^{14}$ However, the electrophysiological substrate is complex and dynamic resulting through an interplay between cardiac anatomy, chamber enlargement from pressure and volume overload, cellular injury from hypoxia and cardiopulmonary bypass, fibrosis at sites of suture lines and patches and direct trauma to the specialised conduction tissue. ${ }^{15}$

As a result, multiple non-clinical tachycardias may be induced at the time of ablation or the tachycardias may not be haemodynamically tolerated to facilitate mapping and ablation. Moreover, it is not uncommon, for no tachycardias to be inducible or sustained or for it to degenerate into atrial fibrillation preventing further mapping to identify the clinically relevant mechanism. All of these could pose potential barriers to acute procedural outcomes and arrhythmia free survival rates post ablation. In our series, no sustained tachycardia could be induced in $32 \%$ of the patients. In these patients we elected to do an empiric CTI line to prevent arrhythmia recurrence. Peri-tricuspid circuits traversing the CTI have been shown to be the single most common mechanism of IART in ACHD patients. ${ }^{16}$ Intuitively; empiric ablation at this site was likely to have a positive impact on preventing arrhythmia recurrence. In keeping with our hypothesis arrhythmia free survival in entrained versus empiric groups were not significantly different $(75 \%$ versus $69 \%, p=0.55)$. One could argue that employing an ablation strategy wherein empiric ablation at sites of slow conduction and low electrogram amplitude could result in incomplete, proarrhythmic lesions and might not always prevent arrhythmia recurrence. It is hard to anticipate how empiric ablation might compare to performing no ablation in patients with complex anatomical substrate and no arrhythmias inducible at the time of the 
procedure. A sizeable proportion of patients fell in this category $\left(1 / 3^{\text {rd }}\right)$ and actually benefitted from having empiric ablation done with the objective end point of arrhythmia free survival.

In the present study, we used arrhythmia free survival as the primary end point to report our ablation success rates. This is largely due to the fact that its is an objective end point that can be easily quantified. However, symptomatic improvement is not driven by complete arrhythmia elimination alone. Reduction in arrhythmia burden and the resulting modification of the haemodynamic substrate in patients with ACHD can significantly improve the morbidity associated with arrhythmias in this population. Perhaps, utilizing an arrhythmia scoring system employing objective and subjective end-points would be a better representation of overall success rates. ${ }^{17}$

The overall arrhythmia free survival rate in our population is not different from what has been reported before. In ACHD patients, most studies have reported arrhythmia recurrence rates of $30-45 \%$ based on complexity of underlying CHD. 5,9 , 17-23 These studies represent a varied arrhythmia substrate in a range of CHD patients but will all ablation carried out to address inducible arrhythmias only. It is interesting to note that this statistic remained the same in the patients undergoing empiric ablation in our study. Moreover, our overall major complication rate was acceptable at $2 \%$ and there was no procedure related mortality. This is at par with what has been reported in this cohort.

In our experience, catheter ablation for IART in ACHD patients is safe and effective. In case of non-inducibility of IART, empiric CTI ablation has good outcomes that are comparable to ablation targeted at inducible arrhythmias. 


\section{STUDY LIMITATIONS}

Some limitations of this study require consideration.

This is a retrospective case-control study. Patients were not randomised to undergo empiric versus entrained CTI line ablation as a first line therapy.

The results of this study reflect the practice of electrophysiologists who are well experienced in performing ablations in patients with complex anatomy. Hence these findings might not be generalizable to other centres, which lack a similar framework. Learning curve of the operator is likely to affect procedural outcomes.

This is a single centre study and sample size is small. The selection of mapping systems, ablation catheters and image integration were operator dependent.

\section{CONCLUSIONS}

Catheter ablation for CTI dependent IART is a safe and effective therapeutic option in patients with ACHD. Long-term arrhythmia free survival after empiric and entrained CTI line ablation are comparable. In case of non-inducibility of IART, an empiric CTI line ablation should be performed in this group of patients.

\section{FUNDING}

This study was not supported by a grant.

\section{ACKNOWLEDGEMENTS}

This study was facilitated by Barts Health NHS Trust. 


\section{REFERENCES}

1. Gewillig M, Cullen S, Mertens B, Lesaffre E, Deanfield J. Risk factors for arrhythmia and death after Mustard operation for simple transposition of the great arteries. Circulation. 1991;84(5 suppl):III187-III192.

2. Gelatt M, Hamilton RM, McCrindle BW, Connelly M, Davis A, Harris

L, Gow RM, Williams WG, Trusler GA, Freedom RM. Arrhythmia and mortality after the Mustard procedure: a 30-year single-center experience. J Am Coll Cardiol. 1997;29:194-201.

3. Gatzoulis MA. Adult congenital heart disease: education, education, education. Nat Clin Pract Cardiovasc Med. 2006;3:2-3.

4. Deal BJ, Mavroudis C, Backer CL. Arrhythmia management in the Fontan patient. Pediatr Cardiol. 2007;28:448-456.

5. Wasmer K, Eckardt L. Management of supraventricular arrhythmias in adults with congenital heart disease. Heart. 2016 Oct 15;102(20):1614-9.

6. Walsh EP. Arrhythmias in patients with congenital heart disease. Card Electrophysiol Rev 2002;6:422-30.

7. Garson Jr A, Bink-Boelkens M, Hesslein PS, et al. Atrial flutter in the young: a collaborative study of 380 cases. J Am Coll Cardiol 1985;6:871-8. 
8. van Hare GF. Radiofrequency ablation of accessory pathways associated with congenital heart disease. Pacing Clin Electrophysiol 1997;20:2077-81.

9. Tanner H, Lukac $P$, Schwick $N$, et al. Irrigated-tip catheter ablation of intraatrial reentrant tachycardia in patients late after surgery of congenital heart disease. Heart Rhythm 2004;1:268-75.

10. Lobo RG, Griffith M, De Bono J. Ablation of Arrhythmias in Patients with Adult Congenital Heart Disease. Arrhythmia \& Electrophysiology Review. 2014;3(1):36-39.

11. Ávila P, Mercier LA, Dore A, et al. Adult congenital heart disease: a growing epidemic. Can J Cardiol 2014;30:S410-19.

12. Somerville J. Management of adults with congenital heart disease: an increasing problem. Annu Rev Med 1997;48:283-93.

13. Bouchardy J, Therrien J, Pilote L, et al. Atrial arrhythmias in adults with congenital heart disease. Circulation 2009;120:1679-86.

14. Walsh EP. Arrhythmias in patients with congenital heart disease. Card Electrophysiol Rev 2002;6:422-30.

15. Walsh EP. Interventional electrophysiology in patients with congenital heart disease. Circulation 2007;115:3224-34.

16. Correa R, Sherwin ED, Kovach J, Mah DY, Alexander ME, Cecchin F, Walsh EP, Triedman JK, Abrams DJ. Mechanism and ablation of arrhythmia following total cavopulmonary connection. Circ Arrhythm Electrophysiol. 2015 Apr;8(2):318-25. 
17. Triedman JK, Alexander ME, Love BA, Collins KK, Berul Cl, Bevilacqua LM, Walsh EP. Influence of patient factors and ablative technologies on outcomes of radiofrequency ablation of intra-atrial re-entrant tachycardia in patients with congenital heart disease. J Am Coll Cardiol.2002;39:1827-1835.

18. Kannankeril PJ, Anderson ME, Rottman JN, Wathen MS, Fish FA. Frequency of late recurrence of intra-atrial re-entry tachycardia after radiofrequency catheter ablation in patients with congenital heart disease. Am J Cardiol 2003;92:879-81.

19. Triedman JK, Bergau DM, Saul JP, Epstein MR, Walsh EP. Efficacy of radiofrequency ablation for control of intraatrial re-entrant tachycardia in patients with congenital heart disease. J Am Coll Cardiol 1997;30:1032- 8.

20. Kalman JM, VanHare GF, Olgin JE, Saxon LA, Stark SI, Lesh MD. Ablation of "incisional” re-entrant atrial tachycardia complicating surgery for congenital heart disease. Use of entrainment to define a critical isthmus of conduction. Circulation 1996;93:502-12.

21. Triedman JK, Saul JP, Weindling SN, Walsh EP. Radiofrequency ablation of intra-atrial re-entrant tachycardia after surgical palliation of congenital heart disease. Circulation 1995;91:707-14.

22. Kanter RJ, Papagiannis J, Carboni MP, Ungerleider RM, Sanders WE, Wharton JM. Radiofrequency catheter ablation of supraventricular tachycardia substrates after Mustard and Senning operations for d-transposition of the great arteries. J Am Coll Cardiol 2000;35:428 - 41. 
23. Nakagawa H, Shah N, Matsudaira K, et al. Characterization of re-entrant circuit in macrore-entrant right atrial tachycardia after surgical repair of congenital heart disease: isolated channels between scars allow "focal" ablation. Circulation 2001;103:699-709. 


\section{TABLES}

Table 1: Baseline Demographics

\begin{tabular}{|l|c|c|c|}
\hline $\mathbf{N}=\mathbf{8 7}$ & EMPIRIC & ENTRAINED & p-Value \\
\hline Number of patients & 28 & 59 & \\
\hline Age $(\mathrm{yrs})$, mean \pm SD & $40 \pm 13.5$ & $46 \pm 16.9$ & 0.10 \\
\hline Gender, male $(\%)$ & 67 & 36 & 0.84 \\
\hline Commonest CHD & ASD $(43 \%)$ & Fallots $(21 \%)$ & 0.85 \\
\hline RA diameter $(\mathrm{mm})$, mean \pm SD & $24 \pm 4.2$ & $24.6 \pm 6.5$ & 0.65 \\
\hline
\end{tabular}

Table 1: Baseline demographics. The Empiric CTI group (Cases) and Entrained CTI group (controls) had 28 and 59 patients respectively. CHD: congenital heart disease; $R A$ : right atrial 
Table 2: Procedural Characteristics

\begin{tabular}{|l|c|c|c|}
\hline $\mathrm{N}=87$ & EMPIRIC & ENTRAINED & p-value \\
\hline Elective (\%) & 80 & 75 & 0.98 \\
\hline General Anaesthetic (\%) & 54 & 61 & 0.96 \\
\hline Procedure time (min), mean \pm SD & $169 \pm 53$ & $183 \pm 57$ & 0.27 \\
\hline Fluoroscopy time (min), mean \pm SD & $28 \pm 31$ & $20 \pm 18$ & 0.13 \\
\hline Procedural Success (\%) & 93 & 95 & 0.99 \\
\hline AAD on follow-up & $0.74 \pm 0.59$ & $0.57 \pm 0.57$ & 0.20 \\
\hline
\end{tabular}

Table 2: Procedural Characteristics. The procedural characteristics of patients undergoing empiric versus entrained CTI line ablation are shown here. AAD: Antiarrhythmic drugs 
Table 3: Multivariate analyses for Predictors of Success

\begin{tabular}{|l|c|c|}
\hline \multicolumn{1}{|c|}{ Variable } & HR & p-value \\
\hline Age, yrs & $0.90(0.81-1.01)$ & 0.09 \\
\hline Gender, male & $0.76(0.26-2.2)$ & 0.63 \\
\hline RA Diameter & $1.1(0.93-1.39)$ & 0.21 \\
\hline Type of CHD & $0.89(0.52-1.55)$ & 0.71 \\
\hline Procedure time & $1(0.99-1.02)$ & 0.72 \\
\hline Ablation Time & $1(0.99-1)$ & 0.96 \\
\hline
\end{tabular}

Table 3: Multivariate analyses for Predictors of Success. A multivariate analyses was carried out to look at the predictors of arrhythmia free survival in the entire cohort. $C H D$ : congenital heart disease; $R A$ : right atrial 


\section{FIGURE LEGENDS}

\section{Figure 1: Underlying Etiology}

Pie chart showing underlying congenital heart disease etiology in the study cohort. $A S D$ : atrial septal defect, VSD: ventricular septal defect, $A V S D$ : atrio-ventricular septal defect, AVR: aortic valve replacement.

\section{Figure 2: Arrhythmia free survival at 20-month follow-up}

Kaplan Myer curves showing arrhythmia free survival up to 20 months of follow-up in patients who had Entrained and Empiric CTI line ablation. There was no significant difference in arrhythmia free survival between the two groups at 20-month follow-up.

\section{Figure 3: Arrhythmia free survival based on underlying etiology}

Bar chart showing arrhythmia free survival at 20-month follow up based on underlying congenital etiology in both the Empiric and Entrained groups. The freedom from arrhythmia post ablation was higher (although not reaching statistical significance) in patients with simple CHD. However, there was no difference in the empiric versus entrained groups based on the underlying etiology ASD: atrial septal defect, ToF: Tetralogy of Fallot 


\section{FIGURES}

Figure 1: Underlying Etiology

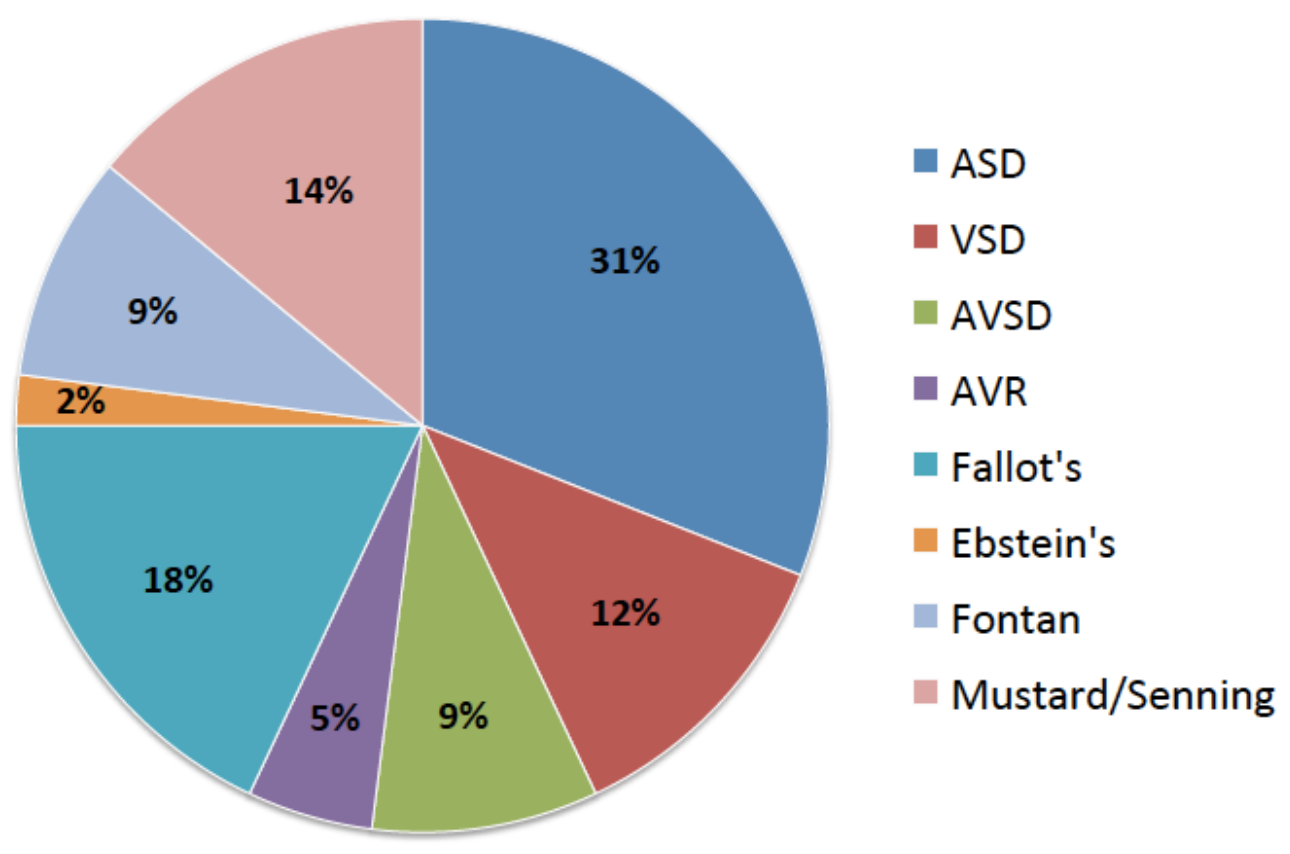


Figure 2: Arrhythmia free survival at 20-month follow-up
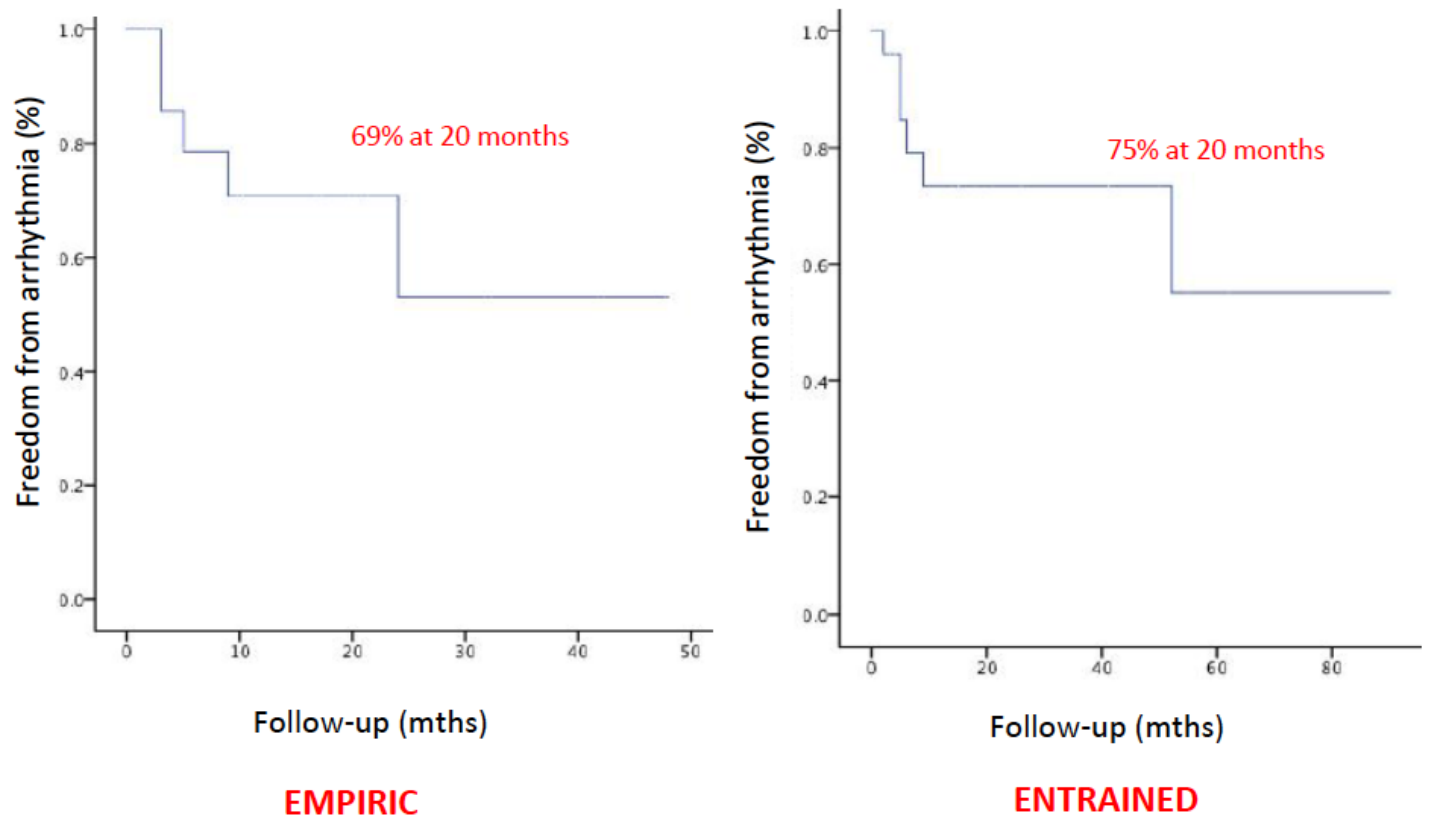
Figure 3: Arrhythmia free survival based on underlying etiology

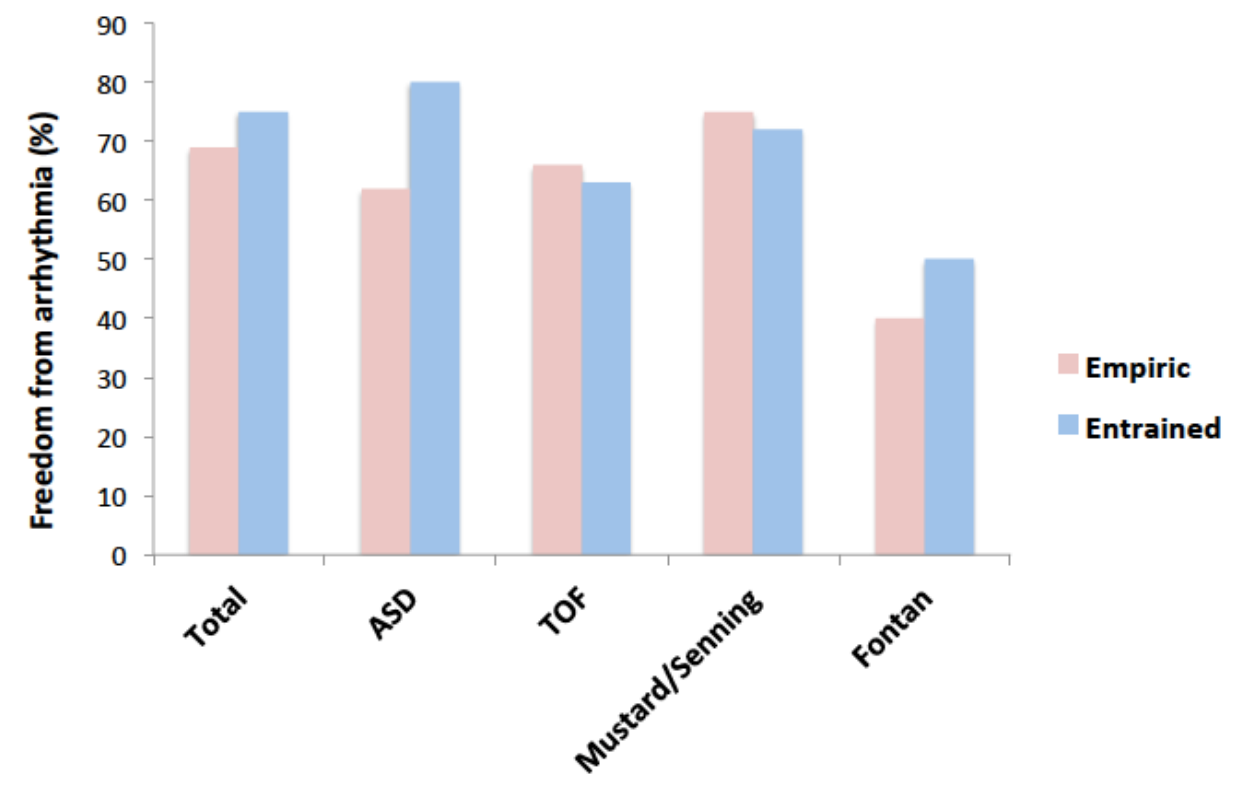

